\title{
Chemical Engineering for Middle School Girls (RESOURCE EXCHANGE)
}

\author{
Ayse Asatekin, Tufts University \\ Ms. Isadora Shamah, Tufts University Center for Engineering Education and Outreach \\ Miss Abigail Anne Klotz \\ Dr. Merredith D Portsmore, Tufts University
}

Dr. Merredith Portsmore is the Director for Tufts Center for Engineering Education and Outreach (www.ceeo.tufts.edu). Merredith received all four of her degrees from Tufts (B.A. English, B.S. Mechanical Engineering, M.A. Education, $\mathrm{PhD}$ in Engineering Education). Her research interests focus on how children engage in designing and constructing solutions to engineering design problems and evaluating students' design artifacts. Her outreach work focuses on creating resources for K-12 educators to support engineering education in the classroom. She is also the founder of STOMP (stompnetwork.org), LEGOengineering.com (legoengineering.com) and the Teacher Engineering Education Program (teep.tufts.edu).

\section{Michael Forte, Tufts University}

Mr. Russell Lincoln Shute,

I am a Technology Education teacher, who teaches classes that introduce students to engineering concepts, at Silver Lake Regional High School, in Kingston, MA. I have a Bachelors from University of Rochester in Chemical Engineering and a Masters in STEM Education from Tufts University. 


\section{Chemical Engineering for Middle School Girls (RESOURCE EXCHANGE)}

This document presents one activity that was done as part of a week-long middle school summer program focused on introducing chemical engineering to female students. The full schedule and set of activities can be found at https://sites.tufts.edu/middleschoolcheme

Designing Spherification Manufacturing Processes

\section{Engineering Challenge for Grades 6-8}

Students will create a spherified product and experiment with different compositions to attain the best properties while keeping track and minimizing resource usage. Students then work with their peers to scale up this process by designing a flowchart and then a process path to produce their spherified product on a large scale.

\section{Motivation}

Chemical engineering is about developing and designing processes that change raw materials into useful products on an industrial scale. They must manage their resources to get the best result while protecting health and safety, and making the smallest environmental impact possible. Chemical engineering is an abstract concept to many, especially younger children; the aim of this activity is to bring it onto a more understandable plane, especially the concept of scale up, and make it tangible for students in a way that isn't intimidating.

\section{Learning Goals}

1. A chemical reaction is a process in which two or more molecules interact and are converted into different molecules by breaking bonds between atoms and forming new ones; the molecules that go in are called the reactants and the molecules that are formed are called the products.

2. Chemical Engineers have to manage their resources so that they attain the best product most efficiently and effectively while watching costs.

3. Scale up is moving a process from lab-scale to industrial or pilot-plant scale.

4. Scale-up is much more complex than just doubling the scale of a process; a chemical engineer cannot take a chemical process in the lab and drop it into a plant by simply proportionally increasing the chemicals and equipment involved.

5. Chemical Engineers have to make design decisions that affect the environment.

\section{Method}

Reverse spherification is used. A sodium alginate bath is premade for students to cut down on time. Students will choose a liquid to spherify and experiment with different compositions of calcium chloride and calcium lactate to produce the most stable product possible. If it is edible, students will have to balance the requirements of a thick membrane for robustness and a thin membrane for palatability. Students will keep track of their "recipes" and costs of "ingredients" in their lab notebooks. Once students have perfected their spherified good, show a "How It's Made" video and walk through the flowchart with students. Have students create flowcharts for their own processes and then give them machinery brochures to buy machines and plan a pilot plant for their spherified good. Step by step instructions are available at https://sites.tufts.edu/middleschoolcheme

\section{Engineering Requirements/Constraints}

- The spherified product must be robust enough to hold in your hands and move around without popping. 
- Edible spherified products must not have very thick membranes that interfere with taste/texture of product

- If prices are assigned to materials, maximize product while minimizing costs

\section{Materials}
- Calcium Chloride
Soap
Machinery Brochures
Calcium Lactate
Lotion
Pictures of machines
Sodium Alginate
Shampoo
Magnetic white boards
Spoons
Bowls
Magnets
Gatorade
Plastic cups
White Board Markers
Chocolate Milk
Measuring spoons
Fake money
Apple Sauce
Pipettes
- Apple Juice
Syringes $(3-5 \mathrm{~mL})$

\section{Rubric for Assessment}

- The spherified product was robust enough to be passed between people

- The cost of the design met the requirements.

- The process plant is feasible and captures the idea of scale up.

- The description and work in the Engineer's Notebook is complete and well reasoned.

\section{Sample Student Work}
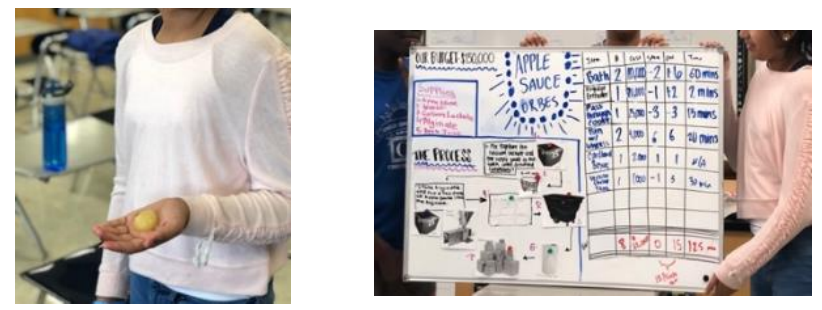

CONTACT INFORMATION: For more information contact ayse.asatekin@ tufts.edu or Merredith.portsmore@tufts.edu. Visit https://sites.tufts.edu/middleschoolcheme

This project was supported by NSF grant \# 1553661 